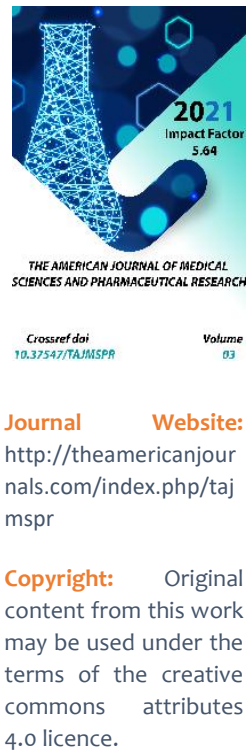

\title{
Etiological Aspects And Preventive Measures Against Chronic Tonsillitis In Children
}

Murodova Mehriniso Mubinovna

Assistant Professor, Department Of Public Health And Health Care Organisation, Tashkent, Uzbekistan

\section{ABSTRACT}

Tonsillitis is a common and frequent disease in childhood, divided into acute tonsillitis and chronic tonsillitis. Acute tonsillitis is caused by pathogens invading the tonsils. Symptoms: fever, cough, sore throat, fever in severe cases, difficulty swallowing, and examination reveals the tonsils are hyperemic, swollen and festering. The tonsils have acute attacks more than 4 times a year, which can be diagnosed as chronic tonsillitis, mostly caused by pathogens in the fossa of the tonsils. On examination, hypertrophy, hyperemia or secretion of the tonsils and enlargement of the submandibular lymph nodes can be seen.

\section{KEYWORDS}

Chronic tonsillitis, tonsils, preventive measures

\section{INTRODUCTION}

Tonsillitis is an inflammation of the tonsils. Clinically, it is divided into acute and chronic. The main symptoms are sore throat, fever and throat discomfort. The condition can cause local or systemic complications, such as ear, nose, heart, kidney and joint complications, so it is worth paying attention to. Acute tonsillitis. Acute non-specific inflammation, 
often secondary to an upper respiratory tract infection, is a very common disease of the throat. It occurs more often in children and young people.

\section{MATERIALS AND METHODS}

The main pathogenic bacteria are betahaemolytic streptococcus, staphylococcus aureus, influenza bacillus or adenovirus, rhinovirus, herpes simplex virus, etc. Mixed infections with bacteria and viruses are also not common. The above-mentioned pathogens exist in the normal oral cavity and tonsils without causing disease [1-3]. When certain factors cause reduced systemic or local resistance, pathogens enter the body or an initial large number of bacteria multiply and cause disease. Cold, damp, excessive fatigue, excessive use of tobacco and alcohol, harmful gases, etc. can be stimuli. Acute tonsillitis is caused by airborne or direct contact. The cause of tonsillitis is related to bacterial and viral infections. The pathogenic bacteria are mainly haemolytic streptococcus, staphylococcus and pneumococcus. These bacteria usually exist in the pharynx and crypts of the human tonsils. Under normal conditions, due to the integrity of the surface epithelium of the tonsils and the constant secretion of mucous glands, the bacteria can be excreted from the crypt openings with the detached epithelial cells, so they remain on the body [4-6]]. When the body's resistance is reduced due to excessive fatigue, cold or local physical or chemical factors, the blood supply to the tonsils decreases, and the secretion function of the glands and the epithelial defence capacity are reduced. Causes the tonsils to become inflamed.

Symptoms are divided into:

- Systemic symptoms occur quickly, headache, chills, high fever can reach 39$40{ }^{\circ} \mathrm{C}$, especially in young children cramps and vomiting may occur because of the high fever;

- The local symptoms of a sore throat are obvious. When the pain is severe, it can cause dysphagia. It may also be recurrent in the ears, tinnitus, a feeling of fullness in the ears and hearing loss. If children have hypertrophy of the tonsils, they may have difficulty sleeping and often wake up at night.

Chronic tonsillitis is most often caused by recurrent episodes of acute tonsillitis or poor drainage of the palatine tonsils, a bacterial viral infection in the fossa and develops into chronic inflammation. It may also be secondary to scarlatina, diphtheria, influenza, measles, infections of the nasal cavity and sinuses. The main pathogenic bacteria are streptococci and staphylococci.

Symptoms: recurrent episodes of acute tonsillitis with throat discomfort and a feeling of blockage. There are also patients who have no history of overt acute attacks and often experience throat discomfort, foreign body sensation, dryness, itching, irritating cough, bad breath, etc. Excessive tonsils can cause difficulty swallowing, slurred speech, difficulty breathing or snoring while sleeping [7-9]]. Chronic tonsillitis is one of the most common diseases. According to a survey, about $22 \%$ of children have chronic tonsillitis.

The tonsils - as we know, they help the body fight infections. They are located on the side wall of the isthmus of the pharynx, between the palatine arch of the tongue and the palatine arch of the pharynx. They have a flatoval shape. The surface is covered with epithelium of the mucous membrane, and the bottom is covered with fibrous membrane. They are loosely connected to the muscular layer of the pharyngeal wall and easily detached; there are pharyngeal tonsils and lingual tonsils. Their main role is in the human 
immune system. They are the largest in the lymphatic tissue of the pharynx and represent a molecule in the lymphatic ring of the pharynx. Between the ages of 6 and 7, the lymphoid tissue grows and the tonsils may become hypertrophied. This is physiological hypertrophy, and after middle age they will gradually shrink and shrink. The main function of these glands is to help the body fight infection, but sometimes it is medically necessary to have them removed. Some people don't want their tonsils removed, even if they have been suffering from chronic inflammation for a long time [10-14]. Is this really a good thing - is it? With tonsillitis, there is an infection of the tonsils. Usually due to a bacterial infection of the throat and upper respiratory tract. In addition, chronic tonsillitis is prone to formation of lesions, allergic reactions and various complications, such as rheumatoid arthritis, rheumatic fever, heart disease, nephritis and other systemic diseases when the internal and external environment of the body changes. Should the tonsils be removed or left behind? Many people have seen only the immune function of the tonsils and would rather suffer repeated tonsillitis than undergo a tonsillectomy [12-15]. This view is wrong: if the tonsils have recurrent inflammation and seriously affect the normal physiological functions of the body, surgical treatment may be considered.

\section{Mostly, tonsils are removed in the following situations:}

1. Repeated episodes of acute tonsillitis, each infection causes fever, sore throat, congestion of the throat mucosa, and at the same time there may be purulent tonsillitis, which is manifested by spots of pus on the surface of the tonsils, which occur more than 4-5 times a year, continuous attacks for 2 years;
2. Excessive hypertrophy of the tonsils makes it difficult to swallow, breathe, vocalise and snore when sleeping;

3. Repeated infections of the tonsils cause rheumatic heart disease, myocarditis, arthritis, glomerulonephritis, skin diseases, etc. The tonsils should be removed;

4. Carriers of diphtheria when conservative treatment is ineffective;

5. Various benign tumours of the tonsils can be removed along with the tonsils.

Preventive measures to prevent chronic tonsillitis in children

Prevention is always the best treatment. To protect your tonsils, you can try the following

1. Exercise more to improve fitness, immunity and resistance to disease;

2. Get plenty of rest and don't overexert your body;

3. Avoid colds and keep away from colds;

4. Drink plenty of water to keep your throat moist;

5. Brush your teeth and rinse your mouth thoroughly to create a favourable environment for the tonsils;

6. Eat less spicy food.

\section{Treatment of tonsillitis in children}

Antibiotic treatment - antibiotics are effective in treating acute tonsillitis and can quickly control symptoms in the acute phase, but they can easily be repeated. Repeated use of antibiotics easily induces drug resistance and can compromise liver and kidney function, lower the immune system and destroy the normal flora of the human body.

The use of lozenges. Tooth lozenges taste good and are easy for patients to take. However, if lozenges are taken regularly, the bacteria will become resistant to the medicine; normal oral bacteria are easily 
damaged and the normal oral flora is disrupted.

Tonsillectomy is performed in recognition of the importance of the tonsils, an immune organ, modern medicine does not support easy removal of the tonsils. If they are removed, they will lose the airway barrier, affecting the entire immune system of the human body. Eliminate surgical treatment.

\section{Topical external treatment}

There are many methods of local treatment of the tonsils. Clinically, these include flushing the crypts of the tonsils, injecting medication into them, local cauterisation, local spraying, laser treatment, etc. Although these methods may inhibit the growth of bacteria and remove lesions, they are more painful.

\section{CONCLUSIONS}

Thus, the tonsils are the gateway to the respiratory tract, and bacteria and viruses in the nose and mouth are the best choice for their invasion. The tonsillar fossa is where bacteria and metabolites accumulate most often. If the humidity and temperature are right, it's easy to get infected. The fossa of the tonsils has a sparse skin, and lymphocytes and other migrating cells are often seen, forming a "fine mesh of fossa epithelium" and becoming the place where the body fights off pathogens and toxins. Because the tonsils have many deep pits, if you suffer from tonsillitis, the pathogen is hidden deep, and it is very easy for lesions to form. When administered orally or intravenously, the effect is not direct and the lesions cannot be completely eliminated. In addition, children's immune systems are not yet normal, so they are vulnerable to repeated attacks from pathogens and recurrent attacks.

\section{REFERENCES}

1. Azimov, M. I., \& Shomurodov, K. E. (2018). A technique for Cleft Palate
Repair. Journal of research in health science, 1(2), 56-59.

2. Garaschenko T.I. (2004). Macrolides in the treatment of acute tonsillitis and its complications in children. Pediatrics. (3). pp. 11-14.

3. Ilyinsky S.E. Mikhailov M.G., Shilenkov A.A., Enin E.A., Kozlov V.S. (2007). Inflammatory diseases of nasopharynx in adults. Russian rhinology. (1). p.16

4. Lopatin A.S. (2002). Acute inflammatory diseases of paranasal sinuses. Reference book of polyclinic doctor. (1). pp. 29-32.

5. A.I. Karpishchenko. (2001). Medical laboratory diagnostics, programs and algorithms. SPb.: Intermedica. p.320.

6. Morozova S.V. (2005). Treatment of acute upper respiratory tract infections. Russian Medical Journal. 13(26(250)). pp.1748-1751.

7. Ovchinnikov A.Y., Gabedava V.A., Ovchinnikov I.A., Syrkin A.L., Svet A.V., Doletsky A.A. (2006). Optimization of treatment of patients with comorbid course of chronic tonsillitis and cardiovascular pathology. Consilium medicum. 8(10). pp. 16-19.

8. Palchun V.T., Luchikhin L.A., Kryukov A.I. (2007). Inflammatory diseases of the pharynx. Moscow: GEOTAR-Media. p.288.

9. Azimov M. I., Shomurodov K.E. (2018). A technique for Cleft Palate Repair. Journal of research in health science. Vol. 1, No. 2, pp. 56-5.

10. Kamalova M. I., Islamov Sh. E., Khaydarov N.K. (2020). Morphological changes in brain vessels in ischemic stroke. Journal of Biomedicine and Practice, vol. 6, issue 5, pp.280-284.

11. Shomurodov. K.E. (2010). Features of cytokine balance in gingival fluid at odontogenicphlegmon of maxillofacial 
area. Doctor-aspirant. 42 Vol.-No.5.1.pp.187-192.

12. Tillyashaykhov M. N., Rakhimov N. M. Khasanov Sh. T., (2019). Features of Clinical Manifestation of the bladder cancer in young people Doctor Bulletin. Samarkand. №2. pp.108-113

13. Ilkhomovna, K. M., Eriyigitovich, I. S., \& Kadyrovich, K. N. (2020). Morphological Features of Microvascular Tissue of The Brain At Hemorrhagic Stroke. The American Journal of Medical Sciences and Pharmaceutical Research, 2(10), 53-59. 